\title{
Bypassing the computational bottleneck of quantum-embedding theories for strong electron correlations with machine learning
}

\author{
John Rogers $\odot,{ }^{1,2}$ Tsung-Han Lee $\odot,{ }^{3}$ Sahar Pakdel $\odot,{ }^{4}$ Wenhu Xu $\odot,{ }^{5}$ Vladimir Dobrosavljević $\odot,{ }^{2}$ Yong-Xin Yao $\odot,{ }^{6}$ \\ Ove Christiansen $\odot,,^{7, *}$ and Nicola Lanatà $\odot^{4,8, \dagger}$ \\ ${ }^{1}$ Department of Physics and Astronomy, Texas A\&M University, College Station, Texas 77845, USA \\ ${ }^{2}$ Department of Physics and National High Magnetic Field Laboratory, Florida State University, Tallahassee, Florida 32306, USA \\ ${ }^{3}$ Physics and Astronomy Department, Rutgers University, Piscataway, New Jersey 08854, USA \\ ${ }^{4}$ Department of Physics and Astronomy, Aarhus University, 8000 Aarhus C, Denmark \\ ${ }^{5}$ Condensed Matter Physics and Materials Science Department, Brookhaven National Laboratory, Upton, New York 11973, USA \\ ${ }^{6}$ Ames Laboratory, U.S. DOE and Department of Physics and Astronomy, Iowa State University, Ames, Iowa 50011, USA \\ ${ }^{7}$ Department of Chemistry, Aarhus University, 8000 Aarhus C, Denmark \\ ${ }^{8}$ Nordita, KTH Royal Institute of Technology and Stockholm University, Roslagstullsbacken 23, 10691 Stockholm, Sweden
}

(Received 9 July 2020; revised 8 December 2020; accepted 12 January 2021; published 29 January 2021)

\begin{abstract}
A cardinal obstacle to performing quantum-mechanical simulations of strongly correlated matter is that, with the theoretical tools presently available, sufficiently accurate computations are often too expensive to be ever feasible. Here we design a computational framework combining quantum-embedding (QE) methods with machine learning. This allows us to bypass altogether the most computationally expensive components of QE algorithms, making their overall cost comparable to bare density functional theory. We perform benchmark calculations of a series of actinide systems, where our method accurately describes the correlation effects, reducing by orders of magnitude the computational cost. We argue that, by producing a larger-scale set of training data, it will be possible to apply our method to systems with arbitrary stoichiometries and crystal structures, paving the way to virtually infinite applications in condensed matter physics, chemistry, and materials science.
\end{abstract}

DOI: 10.1103/PhysRevResearch.3.013101

\section{INTRODUCTION}

The atomic energy scales emerging in "strongly correlated" systems [1-3] can induce a broad spectrum of spectacular effects, ranging from arresting the electronic motion [1] to causing high-temperature superconductivity [4], unlocking access to new topological phases and dramatically influencing the potential-energy surfaces (PES) of molecules and solids [5-10]. Therefore, the need and the potential effects for science and society of extending to strongly correlated systems the computational materials-by-design paradigm can hardly be overstated [2]. The substantial progress achieved in the past decade in calculating the electronic structure of strongly correlated materials is largely owed to the idea of combining mean-field (MF) theories, such as approximations to density functional theory (DFT) [11-17] with quantum-embedding (QE) $[2,18,19]$ theoretical frameworks. Well-known examples are dynamical mean-field theory (DMFT) [20-27] and density matrix embedding theory (DMET) [28,29]. As shown

\footnotetext{
*Corresponding author: ove@ chem.au.dk

${ }^{\dagger}$ Corresponding author: lanata@phys.au.dk
}

Published by the American Physical Society under the terms of the Creative Commons Attribution 4.0 International license. Further distribution of this work must maintain attribution to the author(s) and the published article's title, journal citation, and DOI. in Ref. [30], also the multiorbital Gutzwiller approximation (GA) [30-34], which is a variational framework [equivalent to the rotationally invariant slave boson (RISB) [35-37] at the MF level [38,39]], can be formulated as a QE scheme featuring recursive ground-state calculations of impurity models with a finite bath called "embedding Hamiltonians" (EHs). Therefore, even if the principles underlying $\mathrm{E}=\mathrm{DMFT}, \mathrm{GA}$, RISB, DMET are very different, the concept of QE allows us to formalize and implement these techniques from a unified perspective [30,40,41].

The fundamental idea underlying all QE theoretical frameworks consists in replacing the original (typically unfeasible) problem of directly simulating these systems with the more manageable task of solving equations for a series of EHs, composed by fractions of the material (impurities) and effective-medium degrees of freedom (self-consistently determined for describing the interaction of the impurities with their environment). The current state-of-the-art approach to tackle QE simulations is based on solving the EH equations recursively utilizing many-body techniques [42-45]. On the other hand, due to the quantum-mechanical nature of the $\mathrm{EH}$, its solution ultimately has a computational cost that grows exponentially with the number of impurity degrees of freedom. Because of this reason, the practical application of these tools to complex materials is often too computationally demanding to ever be feasible.

Here we show that this problem can be efficiently tackled from a completely different perspective: capitalizing on the 
fact that the form of the $\mathrm{EH}$ is universal (i.e., it does not depend on the specific stoichiometry and crystal structure of the material considered), we bypass altogether the computationally expensive recursive solution of the EH by "training a machine" to solve this problem once and for all. To accomplish this goal, we develop a computational framework combining machine-learning (ML) techniques, such as 'kernel ridge regression" (KRR), with a mathematical method named "n-mode representation" [46,47]—previously used for effectively reducing the dimensionality of large-scale regression problems (e.g., for reducing the number of points required for constructing high-dimensional PESs in quantum chemistry $[48,49])$.

Note the fundamentally different nature of our method, which employs ML inside the solution of the full quantum problem, with respect to the many current uses of ML for learning preexisting solutions [50] (e.g., for applications to different materials and structures).

We illustrate the power of our method by performing benchmark calculations of a series of actinides. In particular, utilizing our method, we were able to calculate-at a computational cost comparable to bare DFT - the discontinuous behavior of the equilibrium volumes of the actindes as a function of their atomic number $Z$ (actinide transition) [51], which is a phenomenon originated by a complex interplay between structural degrees of freedom, relativistic effects, atom- and orbital-selective electron correlations [30,52-55].

\section{QE ALGORITHMIC STRUCTURE}

The problem of applying QE methodologies (such as the $\mathrm{DFT}+E$ methods) to realistic solids and molecules ultimately reduces to solving recursively multiorbital Hamiltonians represented as follows:

$$
\hat{\mathcal{H}}=\sum_{\mathbf{k}} \sum_{i j=1}^{\eta} \sum_{\alpha=1}^{M_{i}} \sum_{\beta=1}^{M_{j}} \epsilon_{\mathbf{k}, i j}^{\alpha \beta} c_{\mathbf{k} i \alpha}^{\dagger} c_{\mathbf{k} j \beta}+\sum_{\mathbf{R} i} \hat{\mathcal{H}}_{\mathbf{R} i}^{\mathrm{loc}}
$$

where $\mathbf{k}$ is the momentum conjugate to the unit-cell label $\mathbf{R}$, the electronic shells of the atoms within the unit cell are labeled by $i, j=1, \ldots, \eta$, and the corresponding spin orbitals are labeled by $\alpha=1, \ldots, M_{i}, \beta=1, \ldots, M_{j}$. For later convenience, with no loss of generality, we assume that the first term is nonlocal (i.e., that $\sum_{k} \epsilon_{\mathbf{k}, i i}=0 \forall i$ ) and that $\hat{\mathcal{H}}^{\text {loc }}$ includes both the one-body and the two-body local parts of $\hat{\mathcal{H}}$ :

$$
\hat{\mathcal{H}}_{\mathbf{R} i}^{\mathrm{loc}}=\sum_{\alpha \beta=1}^{M_{i}}\left[E_{i}\right]_{\alpha \beta} c_{\mathbf{R} i \alpha}^{\dagger} c_{\mathbf{R} i \beta}+\hat{\mathcal{H}}_{\mathbf{R} i}^{\mathrm{int}}\left[U_{i}, J_{i}\right]
$$

where $E_{i}$ describe the on-site energies [such as the crystalfield energies and the spin-orbit coupling (SOC)] and $\hat{\mathcal{H}}_{\mathbf{R} i}^{\text {int }}$ depends on the Slater-Condon parameters [56], i.e., the Hubbard interaction strength $U_{i}$ and the Hund's coupling constant $J_{i}$.

The basic algorithmic structure of all QE methods to solve the Hamiltonian [Eq. (1)] is schematically illustrated in the left side of Fig. 1. A series of EHs, represented as

$$
\hat{\mathcal{H}}_{\mathbf{R} i}^{\mathrm{emb}}\left[U_{i}, J_{i}, E_{i}, x_{i}\right]=\hat{\mathcal{H}}_{\mathbf{R} i}^{\mathrm{loc}}\left[U_{i}, J_{i}, E_{i}\right]+\hat{\mathcal{B}}_{\mathbf{R} i}\left(x_{i}\right),
$$

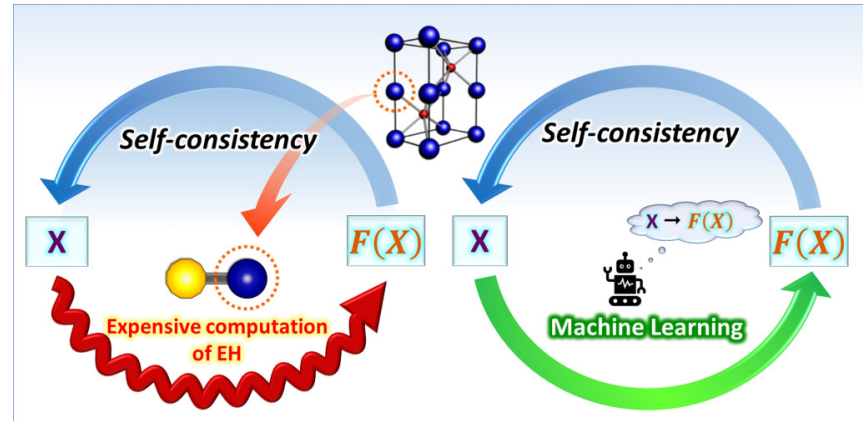

FIG. 1. Algorithmic structure of QE implementations. Standard approach (left): each iteration requires one to solve numerically the EH and calculate the observables $F(X)$ for different descriptors $X$. Proposed approach (right): a ML algorithm, previously trained to learn the universal function $F(X)$, allows us to bypass the computation of the EH.

is constructed for describing the coupling of the impurity with its environment in a MF fashion. Here $\hat{\mathcal{B}}_{\mathbf{R} i}\left(x_{i}\right)$ represents an effective medium coupled with the subsystem (impurity) [Eq. (2)], which is encoded in a series of parameters $x_{i}$. Determining the self-consistent parameters $x_{i}$ requires one to calculate multiple times a series of quantities (varying for different QE methodologies) for the Hamiltonian in Eq. (3), that we schematically represent as $F_{i}$.

For concreteness, here we focus on the GA. As shown in Refs. [30,37] (see also the Supplemental material [57], including Refs. [58,59]), this method can be regarded as a QE framework where

$$
\hat{\mathcal{B}}_{\mathbf{R} i}=\sum_{a \alpha=1}^{M_{i}}\left(\left[\mathcal{D}_{i}\right]_{a \alpha} c_{i \alpha}^{\dagger} f_{i a}+\text { H.c. }\right)+\sum_{a b=1}^{M_{i}}\left[\lambda_{i}^{c}\right]_{a b} f_{i b} f_{i a}^{\dagger},
$$

$\mathcal{D}_{i}, \lambda_{i}^{c}$ are complex $M_{i} \times M_{i}$ matrices encoding the environment of the impurity $i$, the latin labels $a, b$ correspond to the bath degrees of freedom $f$, and the output function $F_{i}$ is the single-particle density matrix:

$$
\left[F_{i}\right]_{A B}=\left\langle\Phi_{i}\left|\left[\psi_{i}^{\dagger}\right]_{A}\left[\psi_{i}\right]_{B}\right| \Phi_{i}\right\rangle
$$

where $\left|\Phi_{i}\right\rangle$ is the ground state of Eq. (3),

$$
\psi_{i}=\left(c_{i 1}, \ldots, c_{i M_{i}}, f_{i 1}, \ldots, f_{i M_{i}}\right)
$$

and the labels $A, B=1, \ldots, 2 M_{i}$ run over both the impurity and the bath degrees of freedom. Therefore, consistently with the general algorithmic structure schematically represented in the left side of Fig. 1, solving the GA equations requires one to evaluate recursively Eq. (5) as a function of the $\mathrm{EH}$ descriptors:

$$
\begin{gathered}
X_{i}=\left(U_{i}, J_{i}, E_{i}, x_{i}\right), \\
x_{i}=\left(\mathcal{D}_{i}, \lambda_{i}^{c}\right) .
\end{gathered}
$$

To simplify the notation, from now on we will omit the electronic-shell label $i$. 


\section{Computational complexity of the EH problem}

In GA $a b$ initio calculations it is typically necessary to deal with impurities consisting of $M=10$ degrees of freedom (for $d$-electron shells) or $M=14$ degrees of freedom (for $f$-electron shells). Since the bath of the EH has the same number of degrees of freedom as the impurity [see Eq. (4)], the dimension of the EH space is $D=2^{2 M}$.

Note that the dimension of the EH system scales as $D=$ $2^{2 M}$ also in DMET. In fact, the differences between these two methods stem exclusively from their different self-consistency conditions [30,40,41]. Within the ghost GA framework (g$\mathrm{GA}$ ), which is a more accurate extension of the GA [34], the number of effective-medium degrees of freedom is still finite, but larger than bare GA. Therefore, the dimension $D$ of the EH system is exponentially higher. Finally, in DMFT [20] the number of effective-medium degrees of freedom (and, therefore, the $\mathrm{EH}$ dimension $D$ ) is infinite.

In all of the theoretical tools mentioned above, the computational bottleneck is solving recursively the EH equations. In fact, this is the only reason why the cost of QE methods generally exceeds by orders of magnitude the cost of meanfield approaches, such as classic approximations to DFT. The computational framework described in the next section will allow us to bypass altogether this problem.

\section{COMBINING ML WITH THE $N$-MODE REPRESENTATION}

Rather than trying to develop more efficient computational tools for solving the EH equations, in this work we will capitalize on the universality of the function [Eq. (5)], utilizing KRR and the $n$-mode expansion for learning it once and for all (see the right side of Fig. 1).

The strategy of utilizing ML for bypassing expensive calculations of universal maps, often referred to as "surrogate modeling," is widely used in physics, chemistry, and materials science [60-66]. The main obstacle to applying classic ML algorithms (such as KRR) for learning multivariable functions is that it requires a number of training data points that scales as

$$
N \sim m^{d}
$$

where $d$ is the number of input variables and $m$ is the number of mesh subdivisions for each dimension. This problem is often referred to as the "exponential curse." Without use of symmetry, the number of input variables in $F(X)$ in Eq. (5) is $d=1+4 M^{2}$, which is 401 for $d$-electron shells and 785 for $f$-electron shells. Therefore, direct applications of ML methods would be extremely costly for learning this function.

To overcome this problem, here we combine KRR with the " $n$-mode representation," which is a technique previously explored in different contexts (and under different names), e.g., for reducing the number of points required to construct high-dimensional PESs $[46,48,49,67]$ and for facilitating the solution of the Schrödinger equation in quantum chemical methods $[68,69]$. The basic idea underlying the $n$-mode representation is to construct approximations to the highdimensional function $F(X)$ in terms of the so-called "cut

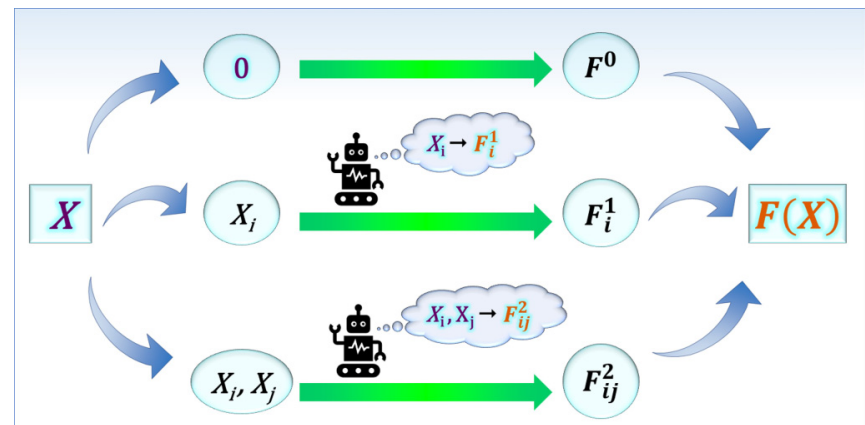

FIG. 2. Representation of the proposed $n \mathrm{KRR}$ approach. The universal $\mathrm{EH}$ function $F(X)$ is approximated with the $n$-mode representation up to the desired order $n$ (e.g., $n=2$ in the picture). The KRR method is used to fit the corresponding lower-dimensional cut functions, which are recombined into an approximation to $F(X)$.

functions," such as

$$
\begin{aligned}
F^{0} & =F(0,0, \ldots, 0,0,0, \ldots, 0,0,0, \ldots, 0), \\
F_{i}^{1}\left(X_{i}\right) & =F\left(0,0, \ldots, 0, X_{i}, 0, \ldots, 0,0,0, \ldots, 0\right), \\
F_{i j}^{2}\left(X_{i}, X_{j}\right) & =F\left(0,0, \ldots, 0, X_{i}, 0, \ldots, 0, X_{j}, 0 \ldots, 0\right),
\end{aligned}
$$

which are the restrictions of $F(X)$ to hyperplanes where subsets of the components of $X$ are set to 0 . At a given order $n$ of the expansion, $F(X)$ is approximated utilizing only cut functions of up to $n$ variables. The exact function is recovered when $n$ equals the total number of variables $d$, and the series converges very rapidly as a function of $n$ in many relevant cases $[46,48,49,67-69]$.

Within our context of application, the main consequence of the $n$-mode expansion is that, since the effective dimensionality is limited to that of the needed order $n$, the input-output mapping can be determined with a number of data points that scales only as

$$
N^{(n)} \sim d^{n},
$$

i.e., it scales polynomially as a function of $d$, rather than exponentially [Eq. (9)]. Specifically, the hereby proposed $n$ KRR methodology consists of the following steps:

(1) Breaking down the universal functions $F(X)$ in lowerdimensional cut functions, using the $n$-mode expansion.

(2) Learning the corresponding lower-dimensional cut functions, up to the desired order, using KRR.

(3) Combining the cut functions into the desired $n$-mode approximation.

A schematic representation of this approach is shown in Fig. 2. For completeness, a short introduction to the KRR method and the $n$-mode representation is provided in the Supplemental Material [57] (including Refs. [70-74]).

We point out that, as opposed to other dimensionalityreduction techniques (where the number of input variables is decreased), the nKRR method allows us to take into account from the outset all descriptors of the EH system, in a manner such that the effective dimensionality is substantially reduced. In the next section we will also capitalize on general physical arguments inherent in the specific structure of Eq. (4). This will allow us to derive a convenient parametrization of the 
EH in DFT+GA calculations, dramatically speeding up the convergence of the $n$-mode representation.

We want to point out that, besides the GA, the nKRR methodology described above could as well be implemented in combination with DMET or more accurate QE methods, such as the g-GA [34] and DMFT.

\section{APPLICATION TO ACTINIDE SYSTEMS}

Here we describe in detail our implementation of the nKRR method for actinide systems. For simplicity, we will focus on the case of a generic EH consisting of $f$-electron shells in an isotropic medium, which is typically a good approximation for actinide systems, where the dominant role of the SOC allows us to average over the crystal-field splittings. Under these assumptions, using group-theoretical considerations, it can be shown [37,75] that the $14 \times 14$ matrices $E, \mathcal{D}, \lambda^{c}$ are diagonal and fully determined by their respective $j=5 / 2$ and $j=7 / 2$ components $E_{j}, \mathcal{D}_{j}, \lambda_{j}^{c}$, where $j$ is the label of the total angular momentum for an $f$-electron shell. Furthermore, as discussed in the Supplemental Material [57], the conservation of the total number of electrons implies that the trace of the single-particle density matrix of the EH is $M$. Therefore, the only independent descriptors of the $\mathrm{EH}$ are the interaction parameters $U, J$ and the following variables:

$$
\begin{aligned}
& X_{1}=\frac{1}{4}\left(E_{5 / 2}+E_{7 / 2}+\lambda_{5 / 2}^{c}+\lambda_{7 / 2}^{c}\right), \\
& X_{2}=\frac{1}{2}\left(E_{5 / 2}-E_{7 / 2}\right), \\
& X_{3}=\frac{1}{2}\left(\lambda_{5 / 2}^{c}-\lambda_{7 / 2}^{c}\right), \\
& X_{4}=\mathcal{D}_{5 / 2}, \\
& X_{5}=\mathcal{D}_{7 / 2} .
\end{aligned}
$$

In fact, with no loss of generality, we can set $\sum_{j \in\{5 / 2,7 / 2\}}\left(E_{j}-\right.$ $\left.\lambda_{j}^{c}\right)=0$, as changing this variable corresponds to applying a chemical-potential shift in Eq. (4) (which would be redundant, as the number of electrons $M$ in the $\mathrm{EH}$ is fixed).

Furthermore, the behavior of $F(X)$ [Eq. (5)] is fully determined by the following functions:

$$
\begin{aligned}
& F_{1}=\frac{1}{4} \sum_{j=5 / 2,7 / 2} \sum_{j_{z}=-j}^{j}\left(\left\langle\hat{c}_{j j_{z}}^{\dagger} \hat{c}_{j j_{z}}\right\rangle-\left\langle\hat{f}_{j j_{z}}^{\dagger} \hat{f}_{j j_{z}}\right\rangle\right), \\
& F_{2}=\frac{1}{2}\left(\sum_{j_{z}=-5 / 2}^{5 / 2}\left\langle\hat{c}_{(5 / 2) j_{z}}^{\dagger} \hat{c}_{(5 / 2) j_{z}}\right\rangle-\sum_{j_{z}=-7 / 2}^{7 / 2}\left\langle\hat{c}_{(7 / 2) j_{z}}^{\dagger} \hat{c}_{(7 / 2) j_{z}}\right\rangle\right), \\
& F_{3}=\frac{1}{2}\left(\sum_{j_{z}=-5 / 2}^{5 / 2}\left\langle\hat{f}_{(5 / 2) j_{z}}^{\dagger} \hat{f}_{(5 / 2) j_{z}}\right\rangle-\sum_{j_{z}=-7 / 2}^{7 / 2}\left\langle\hat{f}_{(7 / 2) j_{z}}^{\dagger} \hat{f}_{(7 / 2) j_{z}}\right\rangle\right), \\
& F_{4}=\frac{1}{2}\left(\sum_{j_{z}=-5 / 2}^{5 / 2}\left\langle\hat{c}_{(5 / 2) j_{z}}^{\dagger} \hat{f}_{(5 / 2) j_{z}}\right\rangle+\sum_{j_{z}=-7 / 2}^{7 / 2}\left\langle\hat{c}_{(7 / 2) j_{z}}^{\dagger} \hat{f}_{(7 / 2) j_{z}}\right\rangle\right), \\
& F_{5}=\frac{1}{2}\left(\sum_{j_{z}=-5 / 2}^{5 / 2}\left\langle\hat{c}_{(5 / 2) j_{z}}^{\dagger} \hat{f}_{(5 / 2) j_{z}}\right\rangle-\sum_{j_{z}=-7 / 2}^{7 / 2}\left\langle\hat{c}_{(7 / 2) j_{z}}^{\dagger} \hat{f}_{(7 / 2) j_{z}}\right\rangle\right),
\end{aligned}
$$

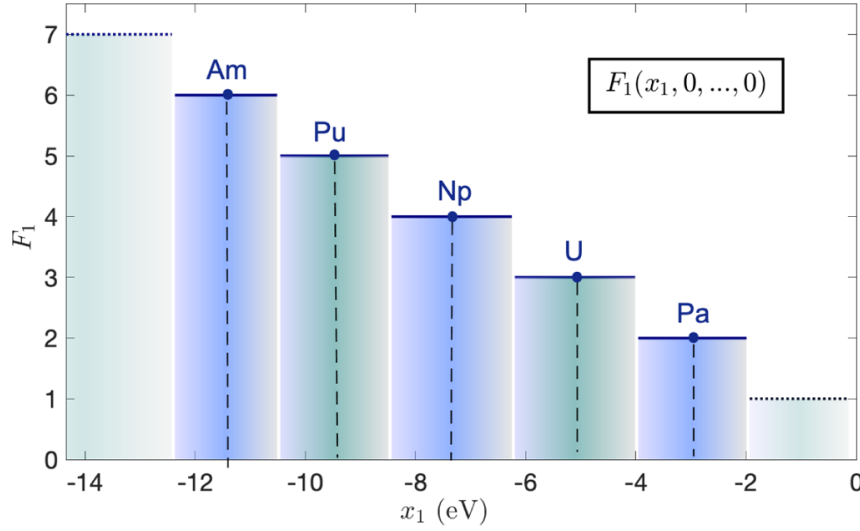

FIG. 3. Behavior of $F_{1}\left(X_{1}, 0, \ldots, 0\right)$, representing the occupation of the impurity for an EH disentangled from the bath site. The mid values of $X_{1}$ of the plateaus are used to set the values of $\bar{x}_{1}$ for all actinides considered.

where $j_{z}$ is the quantum label of the third component of the total angular momentum for each $j$.

Consistently with Ref. [30], here we set the screened Hubbard interaction $U=4.5 \mathrm{eV}$ and the Hund's coupling constant $J=0.36 \mathrm{eV}$. Therefore, the only free embedding descriptors are $X_{1}, \ldots, X_{5}$.

\section{Parametrization of $F(X)$ and training data set}

In this section we describe in detail our procedure for setting up the nKRR method that we are going to utilize for performing DFT + GA calculations of systems involving $\mathrm{Pa}$, $\mathrm{U}, \mathrm{Np}, \mathrm{Pu}$, and $\mathrm{Am}$.

As pointed out in the Supplemental Material [57], the speed of convergence of the $n$-mode representation can be improved by a suitable change of variables, as (by construction) the accuracy of the approximation tends to be higher in the proximity of the domain of the cut functions utilized at the chosen order of truncation. Furthermore, it is pivotal to ensure that the grid of training data points utilized for learning the cut functions [Eq. (10)] is sufficiently large, as ML methods can be predictive only within the training-data range.

It is particularly convenient for our applications to exploit the possibility of expressing the function of Eq. (13) in terms of shifted variables:

$$
Y=X-\bar{X} .
$$

For setting up the components of $\bar{X}$ and the training data set we utilized the following procedure, which is based on physical considerations inherent in the properties of the atomic impurities of interest.

To determine $\bar{X}_{1}$, we have precalculated the behavior of $F_{1}$ as a function of $X_{1}$, at fixed $X_{j}=0 \forall j=2, \ldots, 5$. Note that, in this limit, the impurity $\hat{\mathcal{H}}^{\text {loc }}$ is isolated from the bath. Therefore, the values of $F_{1}$ are quantized and correspond to the nominal (integer) $f$-electron occupations along the actinide series. For each actinide, we have set $\bar{X}_{1}$ as the middle point of the interval of $X_{1}$ values such that $F_{1}\left(X_{1}, 0, \ldots, 0\right)$ equals the corresponding nominal occupation (see Fig. 3). We note that $X_{2}$ describes the impurity SOC, which is essentially an atomic property, i.e., it is typically almost independent of the 

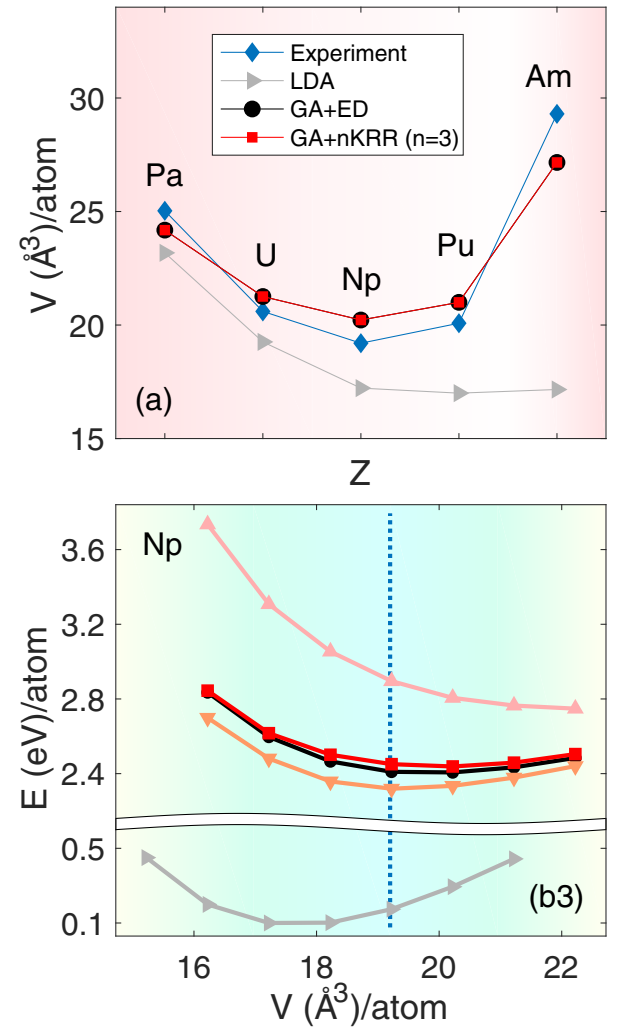
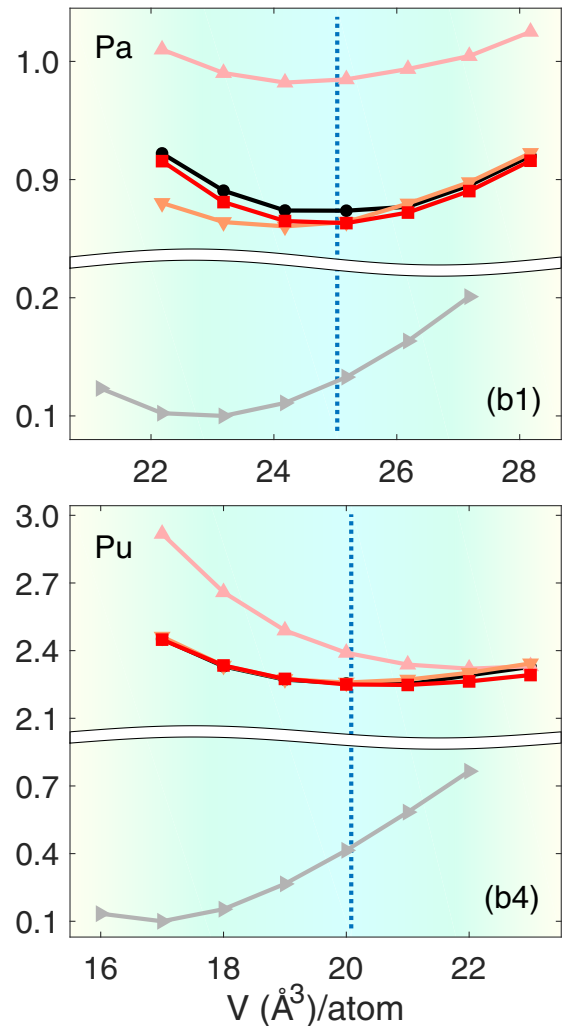
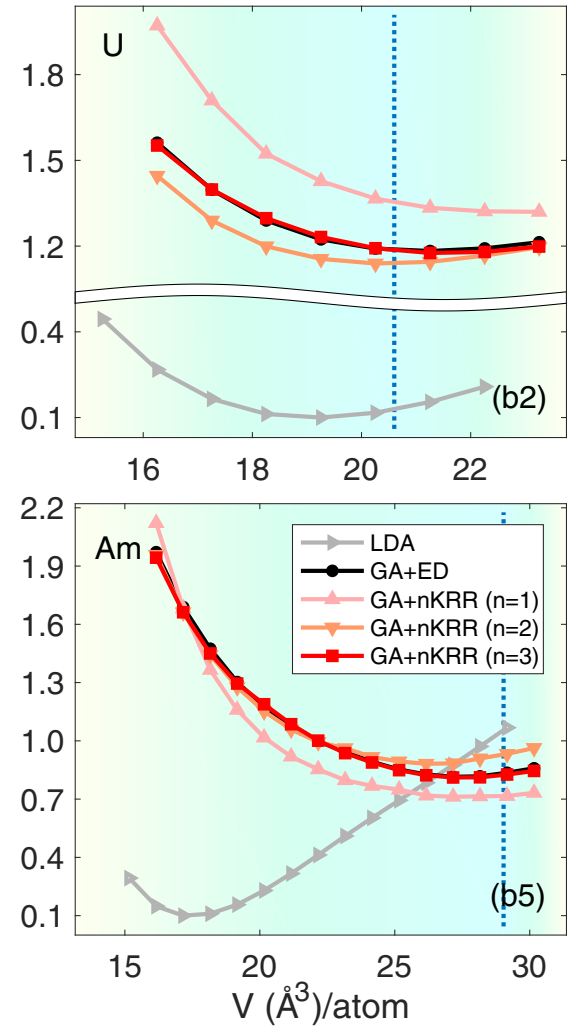

FIG. 4. Panel (a): GA $+n \operatorname{KRR}(n=3)$ and GA+ED equilibrium volumes of the low-temperature allotropes of Pa, U, Np, Pu, Am; in comparison with bare LDA and the experimental values. Panels (b1)-(b5): Corresponding GA $+n K R R(n=1,2,3)$, GA+ED, and LDA energy-volume curves. The vertical blue dashed lines indicate the experimental equilibrium volumes. Within the discrete mesh of volumes considered, the GA+ $n \mathrm{KRR}(n=3)$ and GA+ED minima correspond to the same equilibrium points [panel (a)].

environment in DFT and DFT+GA calculations. Therefore, for each actinide we have set $\bar{X}_{2}$ based on the nominal atomic values, which we precalculated using the local-density approximation (LDA). Since $X_{3}, X_{4}, X_{5}$ describe the EH bath and its coupling with the impurity, their range is generally system dependent. Therefore, the choice of $\bar{X}_{3}, \bar{X}_{4}, \bar{X}_{5}$ is essentially arbitrary. In our calculations we have set them based on a single DFT $+\mathrm{GA}$ calculation of $\delta$-Pu at its experimental equilibrium volume.

The range of the training data set was estimated by performing LDA+GA calculations of $\delta$-Pu at $\pm 35 \%$ of its experimental equilibrium volume. This choice proved to be sufficient for performing all calculations performed in this work. Note that our implementation interactively queries the user if EH parameters beyond the training range are explored in a calculation. Whenever this happens, new training data can be generated and stored in a database. This type of iterative supervised learning, which is often called "active learning procedure," allows one to assess the validity of the simulations and to extend systematically the range of applicability of the nKRR algorithm.

The numerical results reported in the section below were obtained using the following amount of training data for each order of the $n$-mode expansion: 65 data for $n=1,1626$ data for $n=2$, and 19346 for $n=3$. Additional details, such as the numerical values of the components of $\bar{X}$ and the data mesh of the components of $Y$, obtained with the procedure outlined above, are reported in the Supplemental Material [57].

\section{BENCHMARK CALCULATIONS}

To assess the power of our method, we performed LDA+GA benchmark calculations of different actinide solids, utilizing the $n \mathrm{KRR}$ method described above to solve the EH Hamiltonian. We will refer to this framework as the $\mathrm{GA}+n \mathrm{KRR}$, while we will call GA+ED the standard $\mathrm{DFT}+\mathrm{GA}$ approach resulting from using ED as an EH solver.

\section{A. Actinide transition}

A particularly interesting property of the actinide series is the anomalous dependence of their equilibrium volumes as a function of the atomic number. In fact, while the equilibrium volume of the lighter actinides varies continuously as a function $\mathrm{Z}$ (from $\mathrm{Pa}$ to $\mathrm{Pu}$ ), it displays a pronounced discontinuity between $\mathrm{Pu}$ and $\mathrm{Am}$. This volume anomaly is often called actinides transition $[51,76]$, and it is originated by a complex interplay between structural degrees of freedom, SOC, atom- and orbital-selective electron correlations [30,5255]. Therefore, capturing this behavior constitutes a very strict benchmark of our method.

In Fig. 4(a) we show the equilibrium volumes of the low-temperature allotropes of $\mathrm{Pa}, \mathrm{U}, \mathrm{Np}, \mathrm{Pu}$, and $\mathrm{Am}$. The GA+ $+n \operatorname{KRR}(n=3)$ and GA+ED calculations (performed at $U=4.5 \mathrm{eV}$ and $J=0.36 \mathrm{eV}$, as in Ref. [30]) are shown in comparison with LDA and the experiments [52,77]. In Figs. 4(b1)-4(b5) we also show the corresponding 


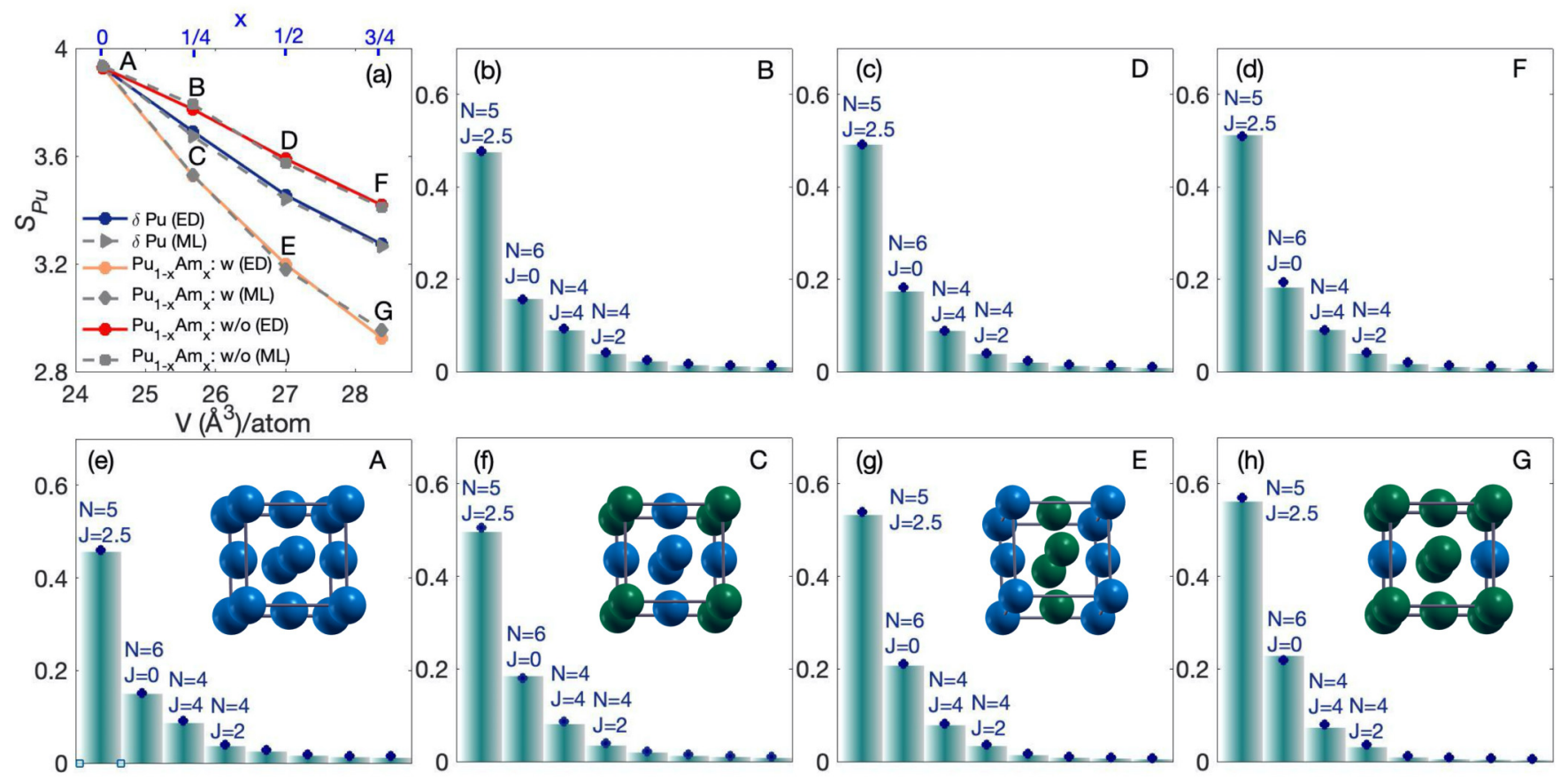

FIG. 5. Comparison between GA+ED and GA+ $n \operatorname{KRR}(n=3)$ calculations of the $\mathrm{Pu}_{1-x} \mathrm{Am}_{x}$ alloys, with $x=0, \frac{1}{4}, \frac{1}{2}, \frac{3}{4}$. (a) Behavior of the entanglement entropy of the $\mathrm{Pu}-5 f$ degrees of freedom. The GA+ ED results obtained by constraining the volume to the experimental value of pure $\delta$-Pu are marked in red, while those obtained varying the volume as a function of $x$, consistently with the experimental expansion $[79,80]$ are marked in yellow, and those for pure $\delta$-Pu are marked in blue. The corresponding results calculated with GA $+n \mathrm{KRR}(n=3)$ are marked in gray. (b)-(h) Eigenvalues of the "reduced density matrix" of a Pu-5f impurity (see the main text) computed with GA+ED (histograms), in comparison with GA $+n \operatorname{KRR}(n=3)$ (blue diamonds). In the pictures of the crystal structures, the Pu atoms are in blue while the Am atoms are in green.

energy-volume curves, whose minima are the points shown in Fig. 4(a). Remarkably, for all systems considered, the GA $+n$ KRR method is substantially more accurate than DFT already for $n=2$, while it becomes essentially as accurate as $\mathrm{GA}+\mathrm{ED}$ for $n=3$.

We point out that each ED solution of the EH Hamiltonian takes about $10 \mathrm{~min}$ and requires $10 \mathrm{~GB}$ of RAM on average, while it takes only about $0.1 \mathrm{~s}$ and $50 \mathrm{MB}$ of RAM within the $n \mathrm{KRR}$ framework. Because of this reason, our GA $+n \mathrm{KRR}$ method is essentially as expensive as bare DFT. In particular, the cost in terms of computational time and RAM is mainly determined by the EH solver within the GA+ED framework. Instead, within GA $+n \mathrm{KRR}$ the computational bottleneck is determined by the DFT operations, such as constructing the Kohn-Sham Hamiltonian and calculating the electron density at each iteration (see the Supplemental Material [57]). For the calculations performed in this work, where the DFT part was performed using the all-electron scheme implemented in WIEN2K [78], utilizing the $n \mathrm{KRR}$ method for solving the $\mathrm{EH}$ equations reduced the computational time by a factor of 10-100 (depending on the system). The relative computational gain of applying our methodology would presumably be even higher by utilizing less computationally demanding implementations of DFT.

\section{B. Local-moment screening in $\mathrm{Pu}_{1-x} \mathrm{Am}_{x}$ alloys}

An important advantage of our computational framework is that it uses ML as a tool for accelerating the simulation itself, rather than for learning the behavior of a single quantity at the time (such as the total energy or the corresponding forces). In fact, since the mathematical structure of the EH (and, in particular, the number of descriptors necessary for specifying it) is identical for homogeneous and heterogeneous systems, the $n \mathrm{KRR}$ predictions are expected to be equally accurate in both cases. Furthermore, once the GA $+n \mathrm{KRR}$ equations are solved, all of the observables accessible to the GA can be computed, simultaneously. To illustrate explicitly these points, in this section we perform benchmark calculations of a series of $\mathrm{Pu}_{1-x} \mathrm{Am}_{x}$ alloys.

Interestingly, in spite of the fact that alloying $\mathrm{Pu}$ with Am results in a considerable volume expansion, the $\mathrm{Pu}$ local moments of these systems remain well screened, indicating that the $\mathrm{Pu}$ degrees of freedom are in a mixed-valent state, as in pure $\mathrm{Pu}$. As previously shown in Ref. [79] utilizing DFT+DMFT, a physical effect hampering the formation of a local moment is that the Am impurities effectively increase the hybridization between the Pu-5 $f$ degrees of freedom near the Fermi level and the itinerant spd electrons.

Here we revisit the analysis of the $\mathrm{Pu}_{1-x} \mathrm{Am}_{x}$ alloys from a DFT+GA perspective, using the $n=3 n \mathrm{KRR}$ method for speeding up the calculations. For simplicity, as in Ref. [79], we consider the $\mathrm{Pu}_{1-x} \mathrm{Am}_{x}$ translationally invariant $f c c$ structures represented in Fig. 5, corresponding to $x=0, \frac{1}{4}, \frac{1}{2}, \frac{3}{4}$. Therefore, we neglect entirely the influence of disorder. In particular, we compare the GA+ED and GA $+n \mathrm{KRR}(n=3)$ calculations for the following cases (see Fig. 5): bare $\delta-\mathrm{Pu}$ at its equilibrium volume (point $\mathrm{A}$ ), a series of $\mathrm{Pu}_{1-x} \mathrm{Am}_{x}$ 
structures at the equilibrium volume of $\delta$-Pu (points B,D,F), and the same structures at the experimental volumes [80] (points C,E,G).

To quantify the influence of the Am doping on the mixedvalent behavior of $\mathrm{Pu}$, we calculate the behavior of the entanglement entropy of the $\mathrm{Pu}-5 f$ degrees of freedom:

$$
S_{\mathrm{Pu}}=\operatorname{Tr}\left[\hat{\rho}_{f}^{\mathrm{Pu}} \ln \left(\hat{\rho}_{f}^{\mathrm{Pu}}\right)\right]
$$

(see Fig. 5), where $\hat{\rho}_{f}^{\mathrm{Pu}}$ is the "reduced density matrix" of a Pu$5 f$ impurity, formally obtained from the GA wave function by tracing out all other degrees of freedom of the system. In Fig. 5 we also show the most relevant eigenvalues of $\hat{\rho}_{f}^{\mathrm{Pu}}$ and the corresponding quantum labels $N$ (number of electrons) and $J$ (total angular momentum).

As expected, the entanglement entropy of $\delta$-Pu decreases monotonically as a function of the volume. In fact, increasing the distance between the atoms amounts to reducing the interatomic interactions. Consistently with previous work $[54,79,81]$, we find that the largest eigenvalue of $\hat{\rho}_{f}^{\mathrm{Pu}}$ is a sixfold degenerate $5 f^{5}$ configuration with total angular momentum $J=\frac{5}{2}$, while the $5 f^{6}$ singlet has a smaller probability weight. On the other hand, the Pu electronic structure remains in a mixed-valent state for all volumes considered, with entanglement entropy substantially larger than $\ln (6) \sim 1.8$ (which is the value that the system would have if the probability weight of the $5 f^{5}$ configuration was 1 ). In agreement with the experiments [82,83], the $\mathrm{Pu}-5 f$ electronic structure remains in a mixed-valent state also in the $\mathrm{Pu}_{1-x} \mathrm{Am}_{x}$ alloys, as the Am atoms induce only minor variations in the $\hat{\rho}_{f}^{\mathrm{Pu}}$ probability weights, and the corresponding entanglement entropy remains much larger than $\ln (6)$ for all of the volumes and Am concentrations considered.

Remarkably, the GA+ED and GA $+n \mathrm{KRR}(n=3)$ calculations of the entanglement entropy and the eigenvalues of $\hat{\rho}_{f}^{\mathrm{Pu}}$ are in very good agreement. We point out that all of the calculations above have been performed using the same $n \mathrm{KRR}$ solver previously constructed for investigating the actinide transition, without any additional training. This confirms that, as expected (because of the general theoretical reasons discussed above), the $\mathrm{QE}+n \mathrm{KRR}$ framework is highly transferable and, in particular, it is equally applicable to stoichiometrically homogeneous and inhomogeneous systems. Therefore, the current implementation of our method can be used to tackle a broad spectrum of important problems, such as accelerating dramatically the molecular-dynamics simulations of real $f$-electron materials, taking into account the strong-correlation effects [84]. Future extensions will allow us to lift the approximation of averaging over the crystal-field splittings, extending even further the range of applicability of our method.

\section{CONCLUSIONS}

In summary, in this work we proposed a computational framework for simulating strongly correlated electron systems, which offers the possibility of substantially stepping up the accuracy with respect to mean-field theories (such as classic approximations to DFT and DFT $+U$ [56]), at a comparable computational cost. This was accomplished by combining QE theoretical frameworks with a fit-for-purpose ML technique (the $n \mathrm{KRR}$ ), where the learning problem is facilitated by the $n$-mode expansion. Based on our results, we argue that our method is already applicable to a broad spectrum of problems inherent in $f$-electron materials, such as dramatically speeding up molecular-dynamics simulations or performing calculations of disordered systems. In fact, these types of calculations require one to solve the EH a very large number of times, which is very problematic with standard tools. The fact that our method reduces the complexity of the learning problem from exponential to polynomial makes it realistically possible to extend it also to systems with arbitrary symmetry, stoichiometry, and crystal structures. Realizing this program will pave the way to virtually infinite applications in condensed matter physics, chemistry, and materials science.

\section{ACKNOWLEDGMENTS}

We thank Gabriel Kotliar for useful discussions and Niels Carl W. Hansen for providing technical support inherent in the computations performed at the Centre for Scientific Computing Aarhus (CSCAA). We gratefully acknowledge funding from VILLUM FONDEN through the Villum Experiment project 00028019 and the Centre of Excellence for Dirac Materials (Grant. No. 11744). We acknowledge support from the Novo Nordisk Foundation through the Exploratory Interdisciplinary Synergy Programme project NNF19OC0057790. T.-H.L. and Y.-X.Y. were supported by the U.S. Department of Energy, Office of Science, Basic Energy Sciences, as part of the Computational Materials Science Program. T.-H.L. was also supported by NSF Grant No. DMR-1733071. Work in Florida was supported by the NSF Grant No. 1822258, and the National High Magnetic Field Laboratory through the NSF Cooperative Agreement No. 1157490 and the State of Florida.

J.R. constructed and trained the $n$ KRR algorithms. J.R., T.-H.L., S.P., and W.X. performed the benchmark calculations of the actinides systems. V.D. cosupervised J.R. and T.-H.L. Y.-X.Y. implemented the $n$ KRR codes within the GA software CyGutz and provided technical support. N.L. and O.C. conceived and led the project. All the authors contributed to the analysis and the interpretation of the results and to writing the manuscript.
[1] N. F Mott, Metal-insulator transitions (Taylor \& Francis, London/Philadelphia, 1990).

[2] P. R. C. Kent and G. Kotliar, Toward a predictive theory of correlated materials, Science 361, 348 (2018).

[3] R. Adler, C.-J. Kang, C.-H. Yee, and G. Kotliar, Correlated materials design: Prospects and challenges, Rep. Prog. Phys. 82, 012504 (2018).
[4] P. A. Lee, N. Nagaosa, and X.-G. Wen, Doping a Mott insulator: Physics of high-temperature superconductivity, Rev. Mod. Phys. 78, 17 (2006).

[5] J. A. Schiller, L. K. Wagner, and E. Ertekin, Phase stability and properties of manganese oxide polymorphs: Assessment and insights from diffusion Monte Carlo, Phys. Rev. B 92, 235209 (2015). 
[6] A. Schrön, C. Rödl, and F. Bechstedt, Energetic stability and magnetic properties of $\mathrm{MnO}$ in the rocksalt, wurtzite, and zincblende structures: Influence of exchange and correlation, Phys. Rev. B 82, 165109 (2010).

[7] J. Kolorenč and L. Mitas, Quantum Monte Carlo Calculations of Structural Properties of FeO Under Pressure, Phys. Rev. Lett. 101, 185502 (2008).

[8] I. Leonov, L. Pourovskii, A. Georges, and I. A. Abrikosov, Magnetic collapse and the behavior of transition metal oxides at high pressure, Phys. Rev. B 94, 155135 (2016).

[9] I. Leonov, V. I. Anisimov, and D. Vollhardt, First-Principles Calculation of Atomic Forces and Structural Distortions in Strongly Correlated Materials, Phys. Rev. Lett. 112, 146401 (2014).

[10] N. Lanatà, T.-H. Lee, Y.-X.Yao, and V. Dobrosavljević, Connection between Mott physics and crystal structure in a series of transition metal binary compounds, npj Comput. Mater. 5, 30 (2019).

[11] P. Hohenberg and W. Kohn, Inhomogeneous Electron gas, Phys. Rev. 136, B864 (1964).

[12] W. Kohn and L. J. Sham, Self-consistent equations including exchange and correlation effects, Phys. Rev. 140, A1133 (1965).

[13] O. Gunnarsson and B. I. Lundqvist, Exchange and correlation in atoms, molecules, and solids by the spin-density-functional formalism, Phys. Rev. B 13, 4274 (1976).

[14] J. P. Perdew, K. Burke, and M. Ernzerhof, Generalized Gradient Approximation Made Simple, Phys. Rev. Lett. 77, 3865 (1996).

[15] R. O. Jones, Density functional theory: Its origins, rise to prominence, and future, Rev. Mod. Phys. 87, 897 (2015).

[16] N. Mardirossian and M. Head-Gordon, Thirty years of density functional theory in computational chemistry: An overview and extensive assessment of 200 density functionals, Mol. Phys. 115, 2315 (2017).

[17] K. Burke, Perspective on density functional theory, J. Chem. Phys. 136, 150901 (2012).

[18] S. Qiming and C. Garnet Kin-Lic, Quantum embedding theories, Acc. Chem. Res. 49, 2705 (2016).

[19] P. Fulde and H. Stoll, Dealing with the exponential wall in electronic structure calculations, J. Chem. Phys. 146, 194107 (2017).

[20] A. Georges, G. Kotliar, W. Krauth, and M. J. Rozenberg, Dynamical mean-field theory of strongly correlated fermion systems and the limit of infinite dimensions, Rev. Mod. Phys. 68, 13 (1996).

[21] V. Anisimov and Y. Izyumov, Electronic structure of strongly correlated materials (Springer, New York, 2010).

[22] G. Kotliar, S. Y. Savrasov, K. Haule, V. S. Oudovenko, O. Parcollet, and C. A. Marianetti, Electronic structure calculations with dynamical mean-field theory, Rev. Mod. Phys. 78, 865 (2006).

[23] K. Held, A. Nekrasov, G. Keller, V. Eyert, N. Blümer, A. K. McMahan, R. T. Scalettar, Th. Pruschke, V. I. Anisimov, and D. Vollhardt, Realistic investigations of correlated electron systems with LDA+DMFT, Phys. Status Solidi B 243, 2599 (2006).

[24] V. I. Anisimov, A. I. Oteryaev, M. A. Korotin, A. O. Anokhin, and G. Kotliar, First-principles calculations of the electronic structure and spectra of strongly correlated systems:
Dynamical mean-field theory, J. Phys.: Condens. Matter 9, 7359 (1997).

[25] T. Maier, M. Jarrell, T. Pruschke, and M. H. Hettler, Quantum cluster theories, Rev. Mod. Phys. 77, 1027 (2005).

[26] M. Potthoff, M. Aichhorn, and C. Dahnken, Variational Cluster Approach to Correlated Electron Systems in Low Dimensions, Phys. Rev. Lett. 91, 206402 (2003).

[27] A. I. Lichtenstein and M. I. Katsnelson, Antiferromagnetism and $d$-wave superconductivity in cuprates: A cluster dynamical mean-field theory, Phys. Rev. B 62, R9283 (2000).

[28] G. Knizia and G. K.-L. Chan, Density Matrix Embedding: A Simple Alternative to Dynamical Mean-Field Theory, Phys. Rev. Lett. 109, 186404 (2012).

[29] I. W. Bulik, G. E. Scuseria, and J. Dukelsky, Density matrix embedding from broken symmetry lattice mean fields, Phys. Rev. B 89, 035140 (2014).

[30] N. Lanatà, Y. X. Yao, C.-Z. Wang, K.-M. Ho, and G. Kotliar, Phase Diagram and Electronic Structure of Praseodymium and Plutonium, Phys. Rev. X 5, 011008 (2015).

[31] M. C. Gutzwiller, Correlation of electrons in a narrow $s$ band, Phys. Rev. 137, A1726 (1965).

[32] J. Bünemann, W. Weber, and F. Gebhard, Multiband Gutzwiller wave functions for general on-site interactions, Phys. Rev. B 57, 6896 (1998).

[33] X.-Y. Deng, L. Wang, X. Dai, and Z. Fang, Local density approximation combined with Gutzwiller method for correlated electron systems: Formalism and applications, Phys. Rev. B 79, 075114 (2009).

[34] N. Lanatà, T.-H. Lee, Y.-X. Yao, and V. Dobrosavljević, Emergent Bloch excitations in Mott matter, Phys. Rev. B 96, 195126 (2017).

[35] R. Frésard and P. Wölfle, Unifieddslave boson representation of spin and charge degrees of freedom for strongly correlated Fermi systems, Int. J. Mod. Phys. B 06, 685 (1992).

[36] F. Lechermann, A. Georges, G. Kotliar, and O. Parcollet, Rotationally invariant slave-boson formalism and momentum dependence of the quasiparticle weight, Phys. Rev. B 76, 155102 (2007).

[37] N. Lanatà, Y. Yao, X. Deng, V. Dobrosavljević, and G. Kotliar, Slave Boson Theory of Orbital Differentiation with Crystal Field Effects: Application to $\mathrm{UO}_{2}$, Phys. Rev. Lett. 118, 126401 (2017).

[38] J. Bünemann and F. Gebhard, Equivalence of Gutzwiller and slave-boson mean-field theories for multiband Hubbard models, Phys. Rev. B 76, 193104 (2007).

[39] N. Lanatà, P. Barone, and M. Fabrizio, Fermi-surface evolution across the magnetic phase transition in the Kondo lattice model, Phys. Rev. B 78, 155127 (2008).

[40] T. Ayral, T.-H. Lee, and G. Kotliar, Dynamical mean-field theory, density-matrix embedding theory, and rotationally invariant slave bosons: A unified perspective, Phys. Rev. B 96, 235139 (2017).

[41] T.-H. Lee, T. Ayral, Y.-X. Yao, N. Lanatà, and G. Kotliar, Rotationally invariant slave-boson and density matrix embedding theory: Unified framework and comparative study on the onedimensional and two-dimensional Hubbard model, Phys. Rev. B 99, 115129 (2019).

[42] J. P. F. LeBlanc, A. E. Antipov, F. Becca, I. W. Bulik, Garnet Kin-Lic Chan, C.-M. Chung, Y. Deng, M. Ferrero, T. M. Henderson, C. A. Jiménez-Hoyos, E. Kozik, X.-W. Liu, A. J. 
Millis, N. V. Prokof'ev, M. Qin, G. E. Scuseria, H. Shi, B. V. Svistunov, L. F. Tocchio, I. S. Tupitsyn, S. R. White, S. Zhang, B.-X. Zheng, Z. Zhu, and E. Gull (Simons Collaboration on the Many-Electron Problem), Solutions of the Two-Dimensional Hubbard Model: Benchmarks and Results from a Wide Range of Numerical Algorithms, Phys. Rev. X 5, 041041 (2015).

[43] K. G. Wilson, The renormalization group: Critical phenomena and the Kondo problem, Rev. Mod. Phys. 47, 773 (1975).

[44] S. R. White, Density Matrix Formulation for Quantum Renormalization Groups, Phys. Rev. Lett. 69, 2863 (1992).

[45] A. Weichselbaum, F. Verstraete, U. Schollwöck, J. I. Cirac, and J. von Delft, Variational matrix-product-state approach to quantum impurity models, Phys. Rev. B 80, 165117 (2009).

[46] S. Carter, S. J. Culik, and J. M. Bowman, Vibrational selfconsistent field method for many-mode systems: A new approach and application to the vibrations of $\mathrm{CO}$ adsorbed on $\mathrm{Cu}(100)$, J. Chem. Phys. 107, 10458 (1997).

[47] Kun Yao, J. E. Herr, and John Parkhill, The many-body expansion combined with neural networks, J. Chem. Phys. 146, 014106 (2017).

[48] K. Emil Lund, T. Bo, G. Ian Heide, and C. Ove, Employing general fit-bases for construction of potential energy surfaces with an adaptive density-guided approach, J. Chem. Phys. 148, 064113 (2018).

[49] K. Carolin and C. Ove, Linear-scaling generation of potential energy surfaces using a double incremental expansion, J. Chem. Phys. 145, 064105 (2016).

[50] J. Schmidt, M. R. G. Marques, S. Botti, and M. A. L. Marques, Recent advances and applications of machine learning in solid-state materials science, npj Comput. Mater. 5, 83 (2019).

[51] R. C. Albers, An expanding view of plutonium, Nature (London) 410, 759 (2001).

[52] B. Amadon, First-principles DFT+DMFT calculations of structural properties of actinides: Role of Hund's exchange, spinorbit coupling, and crystal structure, Phys. Rev. B 94, 115148 (2016).

[53] J.-X. Zhu, R. C. Albers, K. Haule, G. Kotliar, and J. M. Wills, Site-selective electronic correlation in $\alpha$-plutonium metal, Nat. Commun. 4, 2644 (2013).

[54] S. Y. Savrasov, G. Kotliar, and E. Abrahams, Correlated electrons in $\delta$-plutonium within a dynamical mean-field picture, Nature (London) 410, 793 (2001).

[55] L. V. Pourovskii, G. Kotliar, M. I. Katsnelson, and A. I. Lichtenstein, Dynamical mean-field theory investigation of specific heat and electronic structure of $\alpha$ - and $\delta$-plutonium, Phys. Rev. B 75, 235107 (2007).

[56] V. I. Anisimov, F. Aryasetiawan, and A. I. Lichtenstein, Firstprinciples calculations of the electronic structure and spectra of strongly correlated systems: The LDA+U method, J. Phys.: Condens. Matter 9, 767 (1997).

[57] See Supplemental Material at http://link.aps.org/supplemental/ 10.1103/PhysRevResearch.3.013101 for a summary of GA equations, a brief introduction to $n$-mode representation, and details about our implementation of the KRR method.

[58] J. Bünemann, F. Gebhard, and R. Thul, Landau-Gutzwiller quasiparticles, Phys. Rev. B 67, 075103 (2003).

[59] K. Haule, C.-H. Yee, and K. Kim, Dynamical mean-field theory within the full-potential methods: Electronic structure of
$\mathrm{CeIrIn}_{5}, \mathrm{CeCoIn}_{5}$, and $\mathrm{CeRhIn}_{5}$, Phys. Rev. B 81, 195107 (2010).

[60] B. Kanungo, P. M. Zimmerman, and V. Gavini, Exact exchangecorrelation potentials from ground-state electron densities, Nat. Commun. 10, 4497 (2019).

[61] A. P. Bartók, M. C. Payne, R. Kondor, and G. Csányi, Gaussian Approximation Potentials: The Accuracy of Quantum Mechanics, Without the Electrons, Phys. Rev. Lett. 104, 136403 (2010).

[62] C. Nyshadham, M. Rupp, B. Bekker, A. V. Shapeev, T. Mueller, C. W. Rosenbrock, G. Csányi, D. W. Wingate, and G. L. W. Hart, Machine-learned multi-system surrogate models for materials prediction, npj Comput. Mater. 5, 51 (2019).

[63] F. Brockherde, L. Vogt, L. Li, M. E. Tuckerman, K. Burke, and K.-R. Müller, Bypassing the Kohn-Sham equations with machine learning, Nat. Commun. 8, 872 (2017).

[64] L. Li, T. E. Baker, S. R. White, and K. Burke, Pure density functional for strong correlation and the thermodynamic limit from machine learning, Phys. Rev. B 94, 245129 (2016).

[65] Louis-François Arsenault, A. Lopez-Bezanilla, O. A. von Lilienfeld, and A. J. Millis, Machine learning for many-body physics: The case of the Anderson impurity model, Phys. Rev. B 90, 155136 (2014).

[66] K. T. Butler, D. W. Davies, H. Cartwright, O. Isayev, and A. Walsh, Machine learning for molecular and materials science, Nature (London) 559, 547 (2018).

[67] J. O. Jung and R. B. Gerber, Vibrational wave functions and spectroscopy of $\left(\mathrm{H}_{2} \mathrm{O}\right)_{n}, n=2,3,4,5$ : Vibrational selfconsistent field with correlation corrections, J. Chem. Phys. 105, 10332 (1996).

[68] P. M. Zimmerman, Strong correlation in incremental full configuration interaction, J. Chem. Phys. 146, 224104 (2017).

[69] H. Stoll, Toward a wavefunction-based treatment of strong electron correlation in extended systems by means of incremental methods, J. Chem. Phys. 151, 044104 (2019).

[70] H. Stoll, Correlation energy of diamond, Phys. Rev. B 46, 6700 (1992).

[71] J. Friedrich and K. Walczak, Incremental CCSD(T)(F12)|MP2F12-A Mm to obtain highly accurate $\operatorname{CCSD}(\mathrm{T})$ energies for large molecules, J. Chem. Theory Comput. 9, 408 (2013).

[72] I. M Sobol, Global sensitivity indices for nonlinear mathematical models and their Monte Carlo estimates, Math. Comput. Simulat. 55, 271 (2001).

[73] M. Griebel, Sparse grids and related approximation schemes for higher dimensional problems, in Foundations of Computational Mathematics (FoCM05), Santander, edited by L. Pardo, A. Pinkus, E. Suli, and M. J. Todd (Cambridge University Press, Cambridge, UK, 2006), pp. 106-161.

[74] L. Chen, H. Wang, F. Ye, and W. Hu, Comparative study of HDMRs and other popular metamodeling techniques for high dimensional problems, Struct. Multidisc. O. 59, 21 (2019).

[75] N. Lanatà, H. U. R. Strand, X. Dai, and B. Hellsing, Efficient implementation of the Gutzwiller variational method, Phys. Rev. B 85, 035133 (2012).

[76] K. T. Moore and G. van der Laan, Nature of the $5 f$ states in actinide metals, Rev. Mod. Phys. 81, 235 (2009).

[77] W. H. Zachariasen, On the crystal structure of protactinium metal, Acta Crystallogr. 12, 698 (1959).

[78] K. Schwarz and P. Blaha, Solid state calculations using WIEN2k, Comput. Mater. Sci. 28, 259 (2003). 
[79] J. H. Shim, K. Haule, S. Savrasov, and G. Kotliar, Screening of Magnetic Moments in PuAm Alloy: Local Density Approximation and Dynamical Mean Field Theory Study, Phys. Rev. Lett. 101, 126403 (2008)

[80] F. H. Ellinger, K. A. Johnson, and V. O. Struebing, The plutonium-americium system, J. Nucl. Mater. 20, 83 (1966).

[81] J.-X. Zhu, A. K. McMahan, M. D. Jones, T. Durakiewicz, J. J. Joyce, J. M. Wills, and R. C. Albers, Spectral properties of $\delta$-plutonium: Sensitivity to $5 f$ occupancy, Phys. Rev. B 76, 245118 (2007).

[82] N. Baclet, M. Dormeval, L. Havela, J. M. Fournier, C. Valot, F. Wastin, T. Gouder, E. Colineau, C. T. Walker, S. Bremier, C.
Apostolidis, and G. H. Lander, Character of $5 f$ states in the $\mathrm{Pu}$ Am system from magnetic susceptibility, electrical resistivity, and photoelectron spectroscopy measurements, Phys. Rev. B 75, 035101 (2007).

[83] P. Javorský, L. Havela, F. Wastin, E. Colineau, and D. Bouëxière, Specific Heat of $\delta-\mathrm{Pu}$ Stabilized by Am, Phys. Rev. Lett. 96, 156404 (2006).

[84] G.-W. Chern, K. Barros, C. D. Batista, J. D. Kress, and G. Kotliar, Mott Transition in a Metallic Liquid: Gutzwiller Molecular Dynamics Simulations, Phys. Rev. Lett. 118, 226401 (2017). 